\section{Relativity different}

John D. Barrow

General Relativity: An Introduction to the Theory of the Gravitational Field. By H. Stephani.

Cambridge University Press: 1982. Pp.310. £25, \$49.50.

As TIME goes on, it actually becomes easier to write a good textbook on general relativity. You simply follow closely the successful features of the many previous expositors. Some of the most successful relativity textbooks clearly owe much to this type of directed natural selection; with only one or two honourable exceptions, modern texts do not stray very far from the well-worn path found first by Einstein and then mapped by Eddington in his classic The Mathematical Theory of Relativity.

In order to justify its existence, any new book (or, as in this case, any translation of an older book) must display genuinely positive features rather than merely fail to exhibit negative ones. By this criterion alone Hans Stephani's concise text more than justifies its publication. It provides a fuller exposition and wider range of topics than, say, Landau and Lifshitz, whilst providing more insight into their content than purely "algebraic" expositions such as Weinberg's Gravitation and Cosmology. However, unlike the latter, it has little to say about cosmology.

\section{The new synthesis at school}

\section{John Mann}

Guidebook to Organic Synthesis.

By R.K. Mackie and D.M. Smith. Longman: 1982. Pp.332. £9.95, \$19.95.

Fundamentals of Preparative Organic

Chemistry.

By R. Keese, R.K. Müller and

T.P. Toube.

Ellis Horwood/Halsted: 1982. Pp.146.

Hbk $£ 15, \$ 42.95 ; p b k £ 6.50, \$ 19.95$.

Fundamentals of Organic Chemistry.

By T.W. Graham Solomons.

Wiley: 1982. Pp.830.

Hbk £24.80, \$37.20; pbk £12; \$19.30.

ALMOST two decades have passed since E. J. Corey introduced the term "synthon", and discussed methods for the "disconnection" of synthetic targets to provide a "retrosynthetic analysis" of the problem. These ideas have revolutionized the way in which chemists think about synthesis, but little of this has permeated into undergraduate textbooks. Stuart Warren's Designing Organic Synthesis: A Programmed Introduction to the Synthon Approach (Wiley, 1978) provides an excellent account of retrosynthetic analysis,
Stephani provides straightforward and well-organized introductions to many topics normally regarded as beyond the scope of introductory texts. In particular, one finds spinors and tetrads, Lie derivatives, Petrov's classification, plane waves, homogeneous spaces and Bianchi types, the Reissner-Nordstrom metric, together with instructive and non-standard sections on the Cauchy problem and gravitational waves. Also included are some discussions of exact solutions that differ from the usual textbook fodder; for example, the Weyl and axisymmetric vacuum space-times. My only reservations are that the section on the Kerr solution is slightly perfunctory, especially in the light of the space given to other, less important, topics. Also, out of personal prejudice, too little space is allocated to cosmology.

This is an ideal book for a one-term or one-semester graduate course on general relativity, and it also contains material suitable for presentation in undergraduate courses. It is slightly directed towards the mathematician rather than the physicist, but it refrains from the use of exterior calculus and coordinate-free techniques which should make it quite accessible to physicists or astronomers. Whatever their level or background, however, all potential buyers would welcome a cheaper, paperback edition.

John D. Barrow is a Lecturer in Astronomy at the University of Sussex.

but makes no attempt to cover synthetic methods (though publicity for his new book Organic Synthesis: the Disconnection Approach indicates that the remedy is at hand).

Mackie and Smith's new book thus represents a notable advance in the teaching of organic synthesis to undergraduates, since it interweaves synthetic methodology with retrosynthetic analysis.

After a very brief introduction, the authors deal with functionalization of various acyclic and cyclic systems, then move on to functional group interconversions. Most of the methods discussed are standard, but a few more exotic ones are also included. There follow three chapters on carbon-carbon bond formation. The first of these introduces the terms "synthon" and "disconnection" during a discussion of the strategy of synthesis, and then provides a list of key electrophilic and nucleophilic species which can be employed to effect carbon-carbon bond formation. This leads naturally to in-depth accounts of organometallic compounds and of carbanions; in the former chapter there is comprehensive coverage of Grignard reagents and organocopper compounds, and at the end there are a number of synthetic problems with analyses using the synthon/disconnection approach.

Chapter 5 is lengthy and contains an excellent account of the use of stabilized car- banions and related species (including phosphorus and sulphur yields), again concluding with useful problems and analyses. The formation of carbon-hetero bonds is covered next, and then Chapter 7 deals at length with ring-closure and ring-opening. Baldwin's rules are mentioned (probably for the first time in an undergraduate text), and a useful account of heterocyclic ring synthesis is followed by a long section on cycloadditions and electrocyclic processes. Regioselectivity and stereoselectivity are discussed in terms of the frontier orbital approach, but for a more detailed treatment readers should go to the latest edition of Sykes's Guidebook to Mechanism in Organic Chemistry (for review see Nature 295,$487 ; 1982$ ). This strategem is employed throughout the book, and ensures that the flow of the text is not disturbed by complex mechanistic arguments.

Chapters 8 and 9 deal with reduction and oxidation respectively, and all of the common methods are included. Four short chapters are then devoted to protection of functional groups, organoboranes and their transformations, and phosphorus and silicon reagents in synthesis. The information is more than adequate for undergraduate courses, though sulphur and selenium reagents might have been given an additional chapter. The book concludes with six synthetic problems, providing retrosynthetic analyses and an examination of various routes reported in the research literature - lengthy accounts of steroid and peptide synthesis are included.

Throughout the book references and useful notes appear, and lists of further reading are provided. The authors have successfully arranged a marriage of synthesis and retrosynthesis for the undergraduate reader. Only time will tell whether this book or Stuart Warren's new one will be the more successful.

The book by Keese et al. is a useful compendium of basic laboratory methods and techniques, and ranges from essential firstaid and laboratory safety through to hints on the synthesis of radiolabelled compounds. It has valuable sections on the use of the research literature, report writing, purification of solvents, and structure elucidation using spectroscopic methods, and on all of the common methods of extraction and purification. The account is up-to-date (flash chromatography is, for example, included), enlivened by amusing cartoons, and the student edition is excellent value for money.

Finally, the second edition of Solomons's Organic Chemistry was reviewed two years ago (Nature 289, 721; 1981) and his new text is simply a slight condensation of that book. There are 240 or so fewer pages, about half of this being due to the deletion of special topics sections. The new book is, however, cheaper - so you makes your choice and pays your money. $\square$

John Mann is a Lecturer in Organic Chemistry at the University of Reading. 